[0212-7199 (2007) 24: 2; pp 84-86] ANALES DE MEDICINA INTERNA Copyright (C) 2007 ARAN EDICIONES, S.L.

AN. MED. INTERNA (Madrid) Vol. 24, N. $^{\circ} 2$, pp. $84-86,2007$

\title{
Ictus isquémico secundario a mixoma auricular izquierdo
}

\author{
J. NEVADO PORTERO, J. A. SÁNCHEZ, J. G. PAREJA'1, A. J. MANOVEL, \\ M. J. RODRÍGUEZ \\ Servicios de Cardiología y ${ }^{I}$ Cirugía Cardiaca. Hospital Universitario Virgen del Rocío. \\ Sevilla
}

\begin{abstract}
RESUMEN
Presentamos el caso de una mujer de 43 años, ingresada por infarto isquémico en el territorio de la arteria cerebral media izquierda y con antecedentes de episodios ictales. Tras una serie de pruebas se realiza una ecocardiografía que muestra un mixoma auricular izquierdo como etiología del ictus. Ante la necesidad de exéresis quirúrgica, se solicita una resonancia magnética (RNM) cardiaca para obtener información adicional sobre la anatomía y morfología de la masa auricular.

Se exponen las manifestaciones neurológicas del mixoma cardiaco, siendo el accidente cerebrovascular la presentación más frecuente, tal y como ilustra el caso clínico.
\end{abstract}

PALABRAS CLAVE: Mixoma. Ictus isquémico. Tumor cardiaco.
ISCHEMIC STROKE SECONDARY TO LEFT ATRIAL MYXOMA

\section{ABSTRACT}

We present the case of a 43-year-old-woman with history of ictal episodes who was admitted to the hospital after suffering a stroke affecting the left middle cerebral artery territory. After initial evaluation, an echocardiography identified a left atrial myxoma as aetiology of the stroke. In view of the surgical need, we asked for a cardiac magnetic resonance in order to obtain further anatomical information about the atrial mass.

In the present article we review the neurological manifestations of the cardiac myxoma, considering the ischemic cerebrovascular disease as the most frequent presentation, just as this case illustrates.

KEY WORDS: Myxoma. Ischemic stroke. Cardiac tumour.

Nevado Portero J, Sánchez JA, Pareja JG, Manovel AJ, Rodríguez. MJ. Ictus isquémico secundario a mixoma auricular izquierdo. An Med Interna (Madrid) 2007; 24: 84-86.

\section{INTRODUCCIÓN}

El mixoma es una rara causa a considerar de ictus y accidente isquémico transitorio, que afecta fundamentalmente a pacientes jóvenes de sexo femenino (1). La embolización al sistema nervioso central supone alrededor del 50\% de los sucesos embólicos causados por mixomas de aurícula izquierda (AI), en ocasiones representa la primera manifestación sintomática, es más común en el hemisferio izquierdo, puede ser múltiple y masiva y con una instauración del déficit neurológico gradual o repentina (2). El tratamiento es quirúrgico y el pronóstico a largo plazo excelente, si bien, los focos de metástasis viables resultantes de la embolización, así como los aneurismas vasculares secundarios, pueden producir síntomas años después.

\section{CASO APORTADO}

Mujer de 43 años con antecedentes de cefaleas y tabaquismo. Desde hace un año presentaba episodios de visión borrosa, notando en alguna ocasión pérdida visual en hemicampo derecho, acompañándose de cefaleas que cedían tras tratamiento analgésico. Hace nueve meses presentó episodio ictal transitorio hemisférico izquierdo, que desapareció pasados 30 minutos.

En la mañana del presente ingreso notó torpeza en la mano derecha, desviación de la comisura bucal y dificultad para la articulación de la palabra. Por este motivo acudió a urgencias, siendo valorada por el neurólogo que objetivó paresia facial supranuclear derecha y leve disartria, inestabilidad de miembro superior derecho con torpeza para movimientos finos e hipoestesia táctil y algésica facio-braquial derecha. En urgencias se realizó ECG, en ritmo sinusal, TAC craneal que inicialmente no mostró signos de infarto y Doppler de

Trabajo aceptado: 19 de septiembre de 2006 


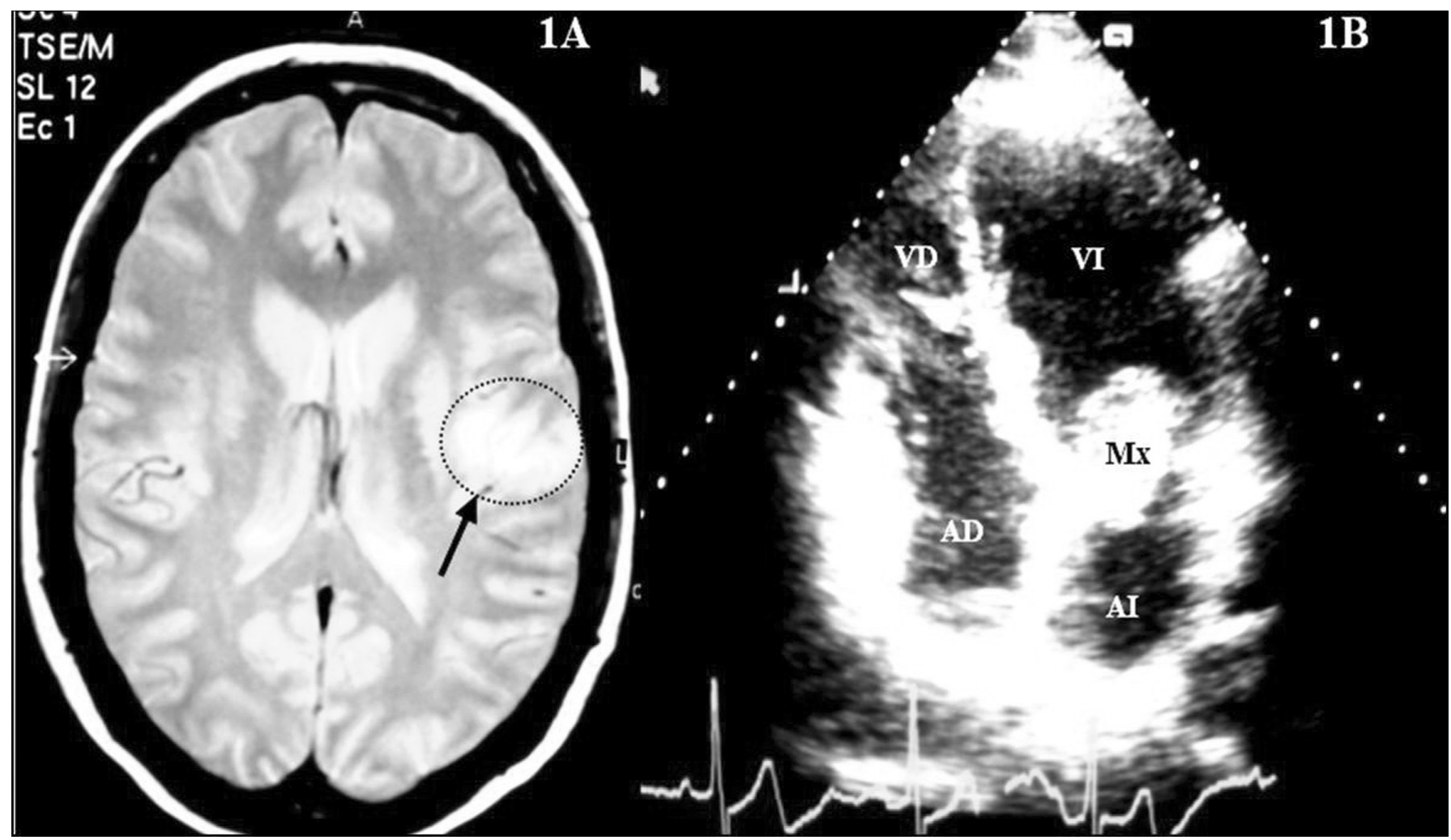

Fig. 1. A: RNM que muestra infarto isquémico en territorio silviano de la arteria cerebral media izquierda (círculo punteado). B: Plano apical cuatro cámaras en diástole con el mixoma prolapsando en ventrículo izquierdo (Al: aurícula izquierda. AD: aurícula derecha. VI: ventrículo izquierdo. VD: ventrículo derecho. Mx: mixoma auricular izquierdo).

troncos supraaórticos y transcraneal que resultaron normales. Se decidió ingreso en planta, solicitándose perfiles analíticos completos incluyendo estudios de hipercoagulabilidad, ANAS, c-ANCA, pANCA, DNA nativo y anticuerpos antimitocondriales. También pruebas de imagen: RNM cerebral y ecocardiografía.

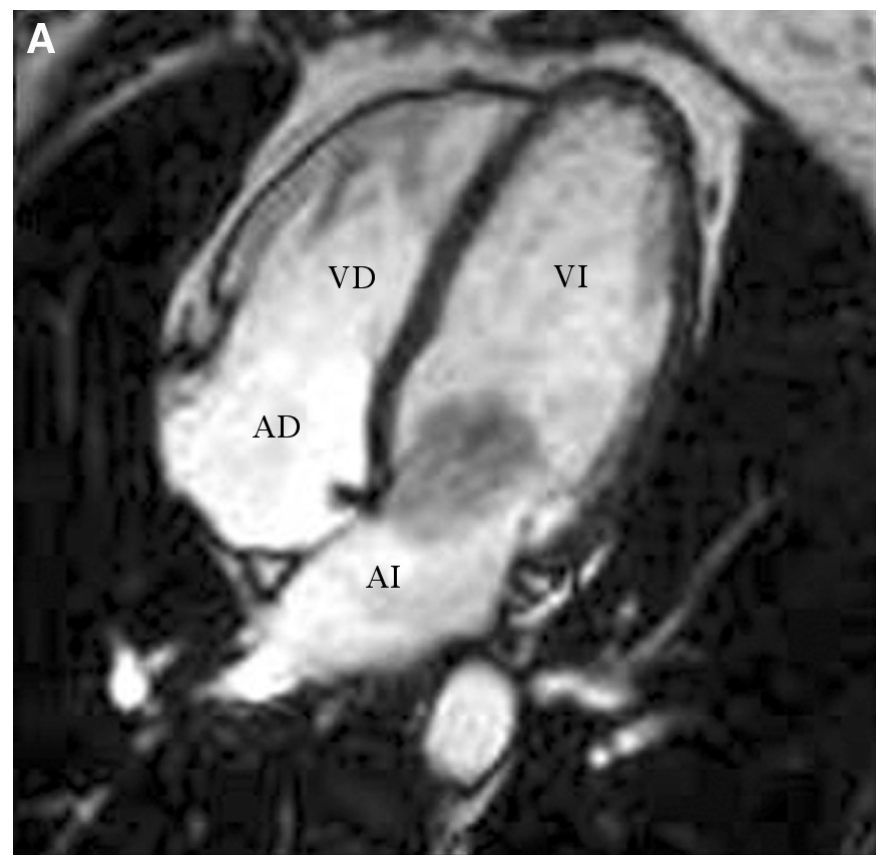

La RNM mostró infarto isquémico agudo en territorio silviano superior de la arteria cerebral media izquierda (Fig. 1A). Los anticuerpos antineutrófilos, anticuerpos antinucleares, anticentrómero y antimitocondrias fueron negativos. El estudio de trombofilia tan sólo detectó ligera hiperfibrinogenemia.

B

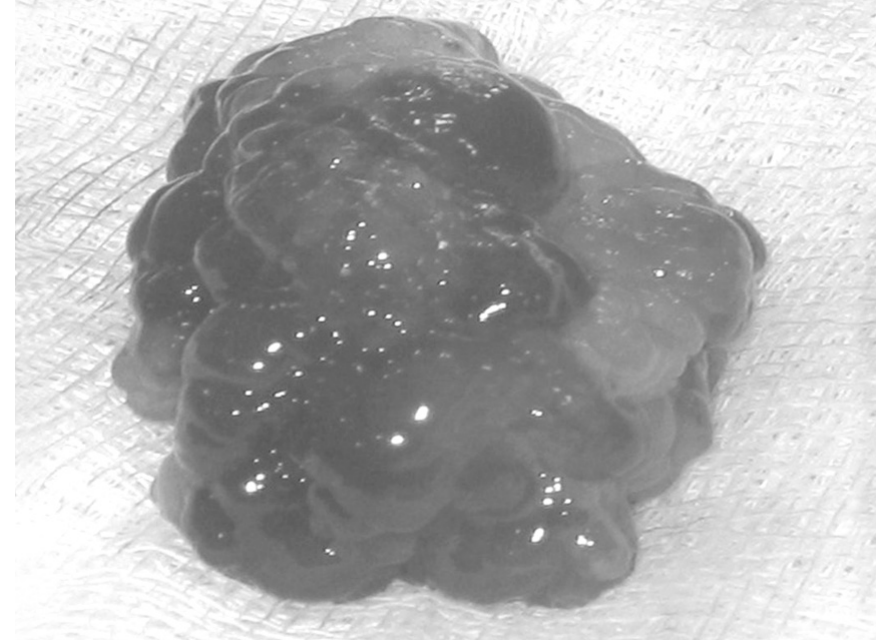

Fig. 2. A: Plano axial 4 cámaras de la RNM cardiaca, que muestra el punto de inserción de la masa auricular en la región límbica de la fosa oval. B: Mixoma auricular extirpado, de consistencia friable y superficie morulada. 
Finalmente se realizó la ecocardiografía, prueba que resultó crucial al proporcionar el diagnóstico definitivo, observándose una masa pedunculada en AI que prolapsaba a través de la válvula mitral en diástole, correspondiente a un mixoma (35 x $26 \mathrm{~mm}$ ), con pedículo en región de la fosa oval (Fig. 1B).

Se programó para cirugía realizándose previamente RNM cardiaca para determinar con mayor precisión las características morfológicas de la masa auricular (Fig. 2A). Se realizó atriotomía izquierda, extrayéndose el tumor en su totalidad, siendo éste de aspecto morulado. Se abrió también aurícula derecha y se resecó la zona de implantación, cerrándose posteriormente la comunicación interauricular creada.

La evolución posquirúrgica resultó muy satisfactoria, siendo dada de alta sin déficit neurológico.

\section{DISCUSIÓN}

Las manifestaciones constitucionales y el potencial embólico son comunes, en distintos grados, en pacientes con mixoma. Un ictus en una persona joven sin evidencia de enfermedad cerebrovascular, especialmente si está en ritmo sinusal, debe llevar a solicitar precozmente pruebas de neuroimagen y ecocardiografía (3), pues hace sospechar la posible presencia de un mixoma intracardiaco en AI, además de un foramen oval permeable, una endocarditis infecciosa o un prolapso de la válvula mitral. Dentro de las manifestaciones neurológicas en pacientes con mixoma la más frecuente es el ictus isquémico $(83 \%)$ frecuentemente en diferentes localizaciones. El resto de síntomas incluye, por este orden, síncope, trastorno psiquiátrico, cefalea y convulsiones (4). No es raro ver en las angiografías múltiples aneurismas vasculares secundarios a embolias tumorales en arterias renales, femorales, coronarias y cerebrales (5) .

Las características de nuestra paciente, mujer joven en ritmo sinusal, con antecedentes de AIT, que ingresa por infarto isquémico de perfil embólico, y en la que el doppler de troncos supraaórticos y transcraneal es normal (lo que descarta inicialmente el foramen oval permeable) son ilustrativas de aquellos casos en los que siempre debe realizarse una ecocardiografía. La RNM se emplea para conseguir una visualización excelente de mixomas intracavitarios, obteniendo información acerca del tamaño, forma, fijación y movilidad de estos tumores (6), lo cual ayuda para la planificación de la resección proporcionando visión tridimensional.

La exéresis quirúrgica es la única terapia aceptable y en los pacientes con antecedentes de embolia, síncopes o evidencias ecocardiográficas de "tumor maligno" (multilobulares, muy grandes, pedículo muy estrecho y protusión constante al ventrículo izquierdo) debe practicarse la cirugía lo antes posible, pues la demora puede llevar a la muerte. Para eliminar completamente un mixoma de la AI se emplea un abordaje biatrial (7), cortando todo el espesor del septum interauricular cuando el tumor está fijado en la región de la fosa oval. El mixoma resecado tenía aspecto morulado, lo cual se asocia de forma significativa a eventos embólicos (8) (Fig. 2B).

La supervivencia tras la resección es excelente, estando recomendado el seguimiento a largo plazo mediante ecocardiografía (9), dado que pueden existir, rara vez, recurrencias del mixoma en un periodo de 48 meses $(10,11)$. Las secuelas neurológicas son poco frecuentes, aunque se han descrito sin recurrencia del tumor primario.

\section{Bibliografía}

1. MacGowan SW, Sidhu P, Aherne T, Luke D, Wood AE, Neligan MC, et al. Atrial Myxoma: national incidence, diagnosis and management. Ir J Med Sci 1993; 162: 223-6.

2. Browne WT, Wijdicks EF, Parisi JE, et al. Fulminant Brain necrosis from atrial myxoma showers. Stroke 1993; 24: 1090-1102.

3. Hart RG, Albers GW, Koudstaal Pj. Cardioembolic stroke. In: Ginsberg MD, Bogouslavsky J, editors. Cerebrovascular Disease: pathophysiology, diagnosis and management. London: Blacwell Science; 1998. p. $1392-429$.

4. Ekinci E, Donnan GA. Neurological manifestations of cardiac myxoma: a review of the literature and report of cases. Int Med J 2004; 34 : 243-249.

5. Michael AS, Mikael MA, Christ M. Myxoma of the Heart presenting with recurrent episodes of hemorrhagic cerebral infartion: MR findings. J Comput Assit Tomogr 89; 13: 123.
6. Matsuoka H, Hamada M, Honda T, et al. Morphologic and histologic characterization of cardiac myxomas by magnetic resonance imaging. Angiology 1996; 47: 693-698.

7. Moreno F, Lagomasino A, Mirabal R, López F, López O. Mixoma Auricular izquierdo recidivante. Archivos de cardiología de México 2003; 4: 279-283.

8. Jones DR, Warden HE, Murray GF, Hill RC, et al. Biatrial approach to cardiac myxomas: A 30-year clinical experience. Ann Thorac Surg 1995; 59: 851-856.

9. Hiroyuki K, Tamotsu Y, Hiroshi N, et al. Surgical Treatment of Primary Cardiac Tumors. Jpn Cir J 2001; 65: 315-319.

10. Leonard M S. Cardiac tumours: diagnosis and management. Heart 2001; 85: 218-222.

11. Roeltgen DP, Weimer GR, Patterson LF. Delayed neurologic complications of left atrial myxoma. Neurology 1991; 31: 8-13. 